\title{
Reference ranges for IgG subclasses in preschool children
}

\author{
D BIRD, S DUFFY, D ISAACS, AND A D B WEBSTER \\ Divisions of Immunological Medicine and Computing and Statistics, Clinical Research Centre, Harrow
}

SUmmary A normal range for IgG1, IgG2, and IgG3 subclasses has been established for children aged 6 months to 5 years using commercially available monoclonal antisera. Of particular interest were the very low concentrations of IgG2 in some healthy children, a finding which casts doubt on the importance of IgG2 deficiency reported in some patients with otherwise unexplained infections. It was not possible to construct a normal range for IgG4 values probably because these segregate into two populations in normal subjects.

There are four known subclasses of IgG (assigned $1-4)$ which constitute approximately $61,30,5$, and $4 \%$ of the total IgG respectively. ${ }^{1}$ Schur et al ${ }^{2}$ were the first to describe patients with recurrent infections who lacked some IgG subclasses while retaining others. This was an important observation because these patients improved on gammaglobulin replacement treatment. Oxelius ${ }^{3}$ described similar patients, and more recently the same group have drawn attention to an association between IgG2 deficiency and selective IgA deficiency, ${ }^{4}$ particularly in patients with ataxia telangiectasia. ${ }^{5}$ It is suggested that those patients with selective IgA deficiency who also lack IgG2 are particularly prone to respiratory infections, although there are exceptions. ${ }^{6}$

Population screening for IgG subclass deficiencies has been hampered by the scarcity of specific antisera. Monoclonal antisera are, however, now available, which should enable us to screen larger populations of patients and obtain a wider view of the importance of these abnormalities; but accurate normal values are needed before this can be achieved. Because children are most likely to present with recurrent infections due to IgG subclass deficiencies, we have produced a normal range for these proteins based on a large group of healthy children.

\section{Materials and methods}

Subjects. Two hundred and fifteen children between the age of 6 months and 6 years gave blood samples, when they attended local child welfare clinics. Informed consent was obtained from the parents. This work was part of a larger study to determine the range of immunoglobulin and anti-pertussis antibody values in healthy children. ${ }^{7}$

IgG subclass measurements. A Mancini technique using commercially available monoclonal IgG subclass antisera (Seward - BAM 09, BAM 10, BAM 08 for IgG 1-3 respectively; both BAM 16 and BAM 11 for IgG 4) was used. Briefly, a normal serum at six different dilutions and the test sera at a fixed dilution were applied to wells cut in agarose gel $(1.4 \%$ in $0.1 \mathrm{M}$ barbitone buffer $\mathrm{pH} 8.6)$ which contained $6 \%$ polyethylene glycol 3000 and monoclonal antiserum. Two different monoclonal antisera were used to measure IgG 4 subclass values because a single antiserum failed to give adequate precipitation. A pooled normal serum was used as a standard which was calibrated against the World Health Organisation (WHO) reference serum (67/97) by comparing four different dilutions $(80,60,40$, and $20 \%$ ) of the latter with the same dilutions of the former. The error for each of these comparisons was within $5 \%$. The concentration of subclasses in the WHO serum had previously been calibrated by Morell and Skvaril. ${ }^{8}$

The gels were left for three days at $4^{\circ} \mathrm{C}$ on a level plate before being photographed against a dark background. A standard curve was plotted on semilog paper of the diameter of the precipitin ring against serum dilution, and the unknowns read off and actual concentration calculated. Serum $\operatorname{IgE}$ was measured by radioimmunoassay ${ }^{9}$ in 58 sera taken at random.

Statistical analysis. Statistical analysis broadly followed the method described by Isaacs et al. Briefly, 
the appropriate polynomial regression model was fitted to the data and the residuals tested for normality. If a significant departure from normality was observed, a suitable transformation was sought. Thus a polynomial regression of mean IgG subclass value on age was obtained, satisfying the normality assumptions and giving an expected value which changed smoothly with age. For standard deviations, the age range was split into six groups $(0-1$, $1-2,2-3,3-4,4-5$, and 5-6 years). Polynomial regression of SD on age was then performed. The reference ranges are given by the fitted expected levels $\pm 1.96 \times$ the fitted SDs, subsequently back transformed where appropriate.

\section{Results}

Normal ranges were found for the IgG subclasses 1-3 (Figs. 1, 2, 3). IgG1 values were found to conform closely to a normal distribution, so no transformation was necessary. Immunoglobulin G2 and IgG3 were approximately log normally distributed, so means and SDs of the logged data were fitted. The fitted polynomial regression equations are given in the appendix. All quoted coefficients were significant at $\mathbf{P}<0 \cdot 0001$. No transformation

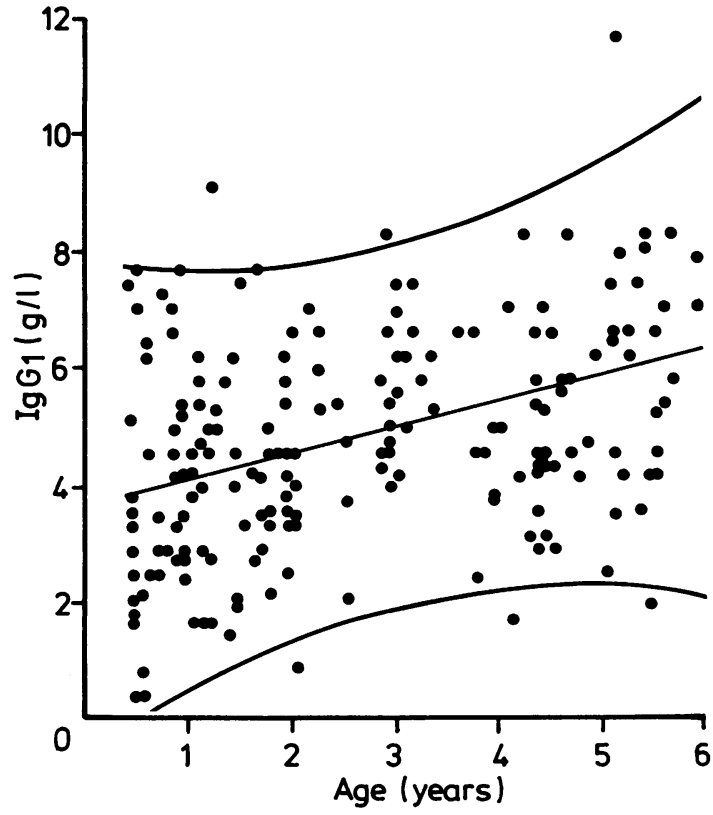

Fig. 1 Scatter diagram of IgGl concentration against age, with age specific mean and $95 \%$ reference range.

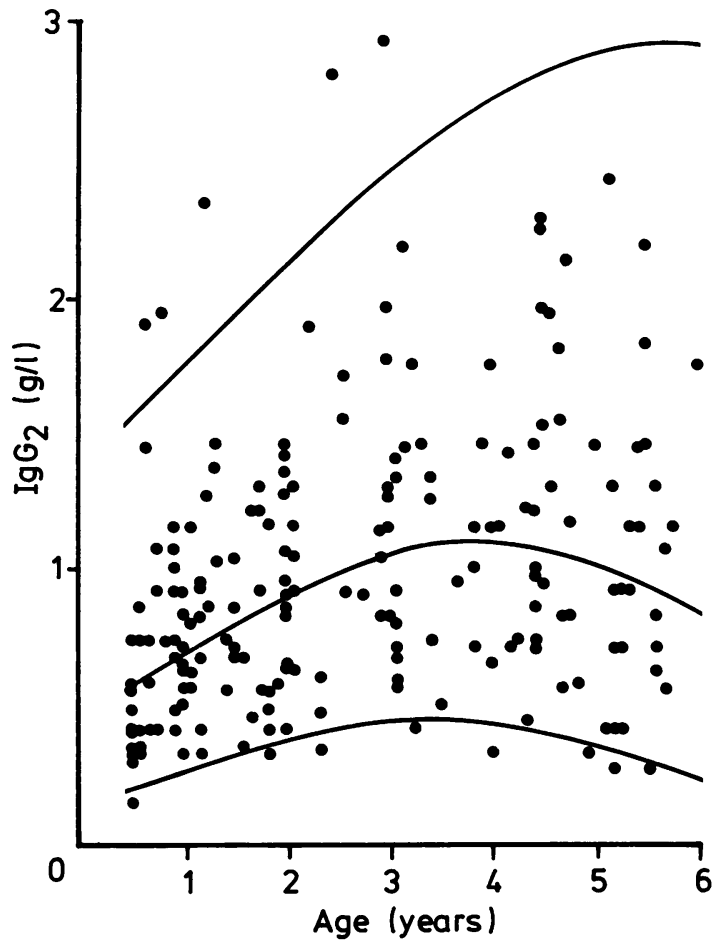

Fig. 2 Scatter diagram of IgG2 concentration against age, with age specific mean and $95 \%$ reference range.

was found which would render the IgG4 data approximately normal, leading us to suspect that we are dealing with more than one population (Fig. 4). Splitting the population into two groups in various ways, however, yielded no improvement. Consequently no reference range for IgG4 has been produced. Fig. 5 shows a significant correlation between IgG4 and IgE values; however, the partial correlation of the two after adjustment for age is $0 \cdot 11$, indicating that this is not likely to be a direct effect.

\section{Discussion}

There is general agreement between the normal ranges provided here and those already published in young children, ${ }^{10-12}$ although there are discrepancies, particularly for the lower limits. This is probably due to inadequate numbers of subjects in these previous studies, or to their expressing the data as means (SD), or both. For accuracy, the normal values presented here should be used for frozen serum samples, since it is known that total IgG concentrations, measured by nephelometry, 


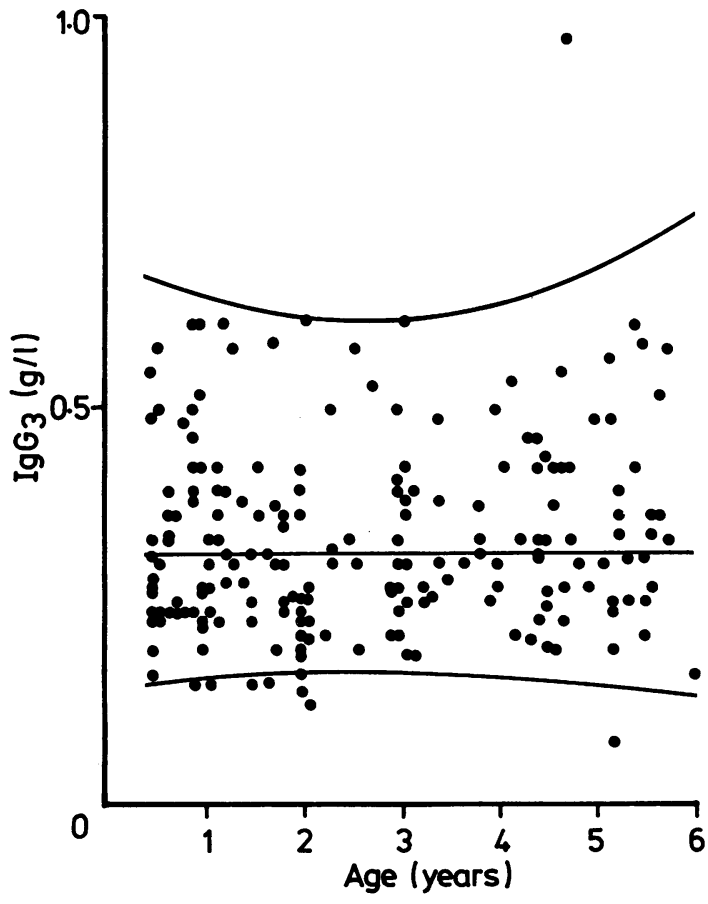

Fig. 3 Scatter diagram of IgG3 concentration against age, with age specific mean and $95 \%$ reference range.

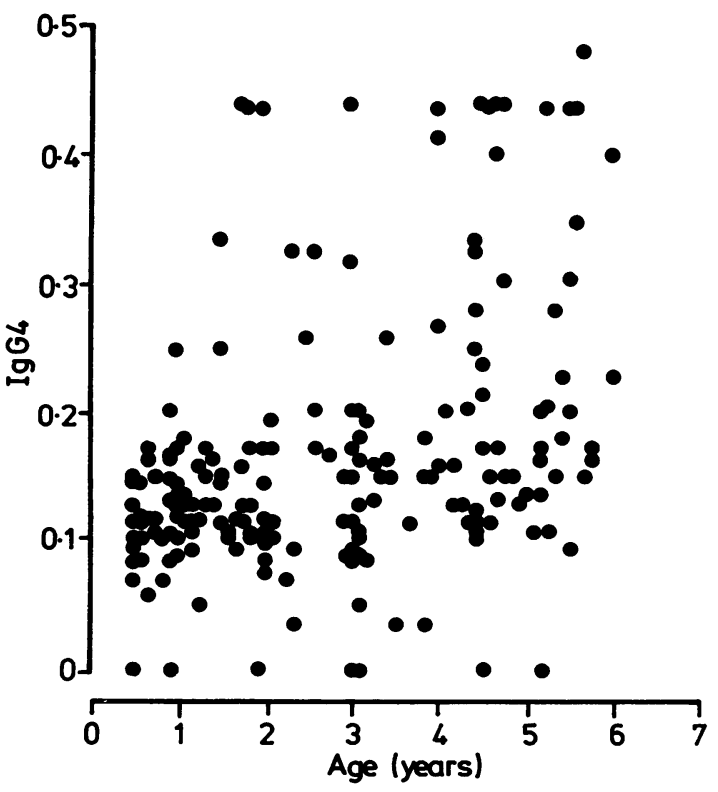

Fig. 4 Scatter plot of IgG4 concentrations against age.

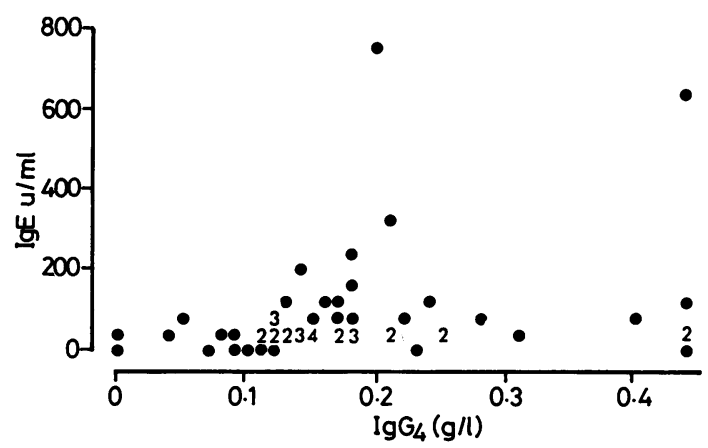

Fig. 5 Scatter diagram of IgE concentration against IgG4 for 58 children drawn randomly from the 215 subjects.

Spearman's rank correlation coefficient $=0.31 \quad(P=0.02$ approximately $)$.

may not give the same readings when stored frozen sera are compared with sera stored at $4^{\circ} \mathrm{C} .{ }^{13}$

The ranges reported here differ from those recently published for English adult subjects using the same monoclonal antisera. ${ }^{14}$ This confirms previous studies which show that there is a further rise in all subclasses between the age of 6 and 20 years. ${ }^{1011}$

The IgG2 values are particularly interesting as they are much lower in young children than in adults. Although $\mathrm{IgG}$ antibodies to techoic acid and some polysaccharides ${ }^{1516}$ seem to be confined to the IgG2 subclass, the clinical importance of IgG2 deficiency is not yet clear. At all events, it is not uncommon for healthy children at 6 months to have IgG2 values of about $0 \cdot 2 \mathrm{~g} / \mathrm{l}$. Such children usually also have very low serum IgA values. ${ }^{7}$ This suggests that IgG2 antibodies are not critical for host defence, and a deficiency of this subclass probably has to be accompanied by other defects before overt disease develops.

Wide variations are seen in the IgG4 concentrations, preventing a normal range being constructed. A similar problem is encountered with IgE values in both adults and children, and it is interesting that we found a significant association between serum $\mathrm{IgG} 4$ and IgE concentrations in our study. This apparent association may not be direct, however, and is probably due to a correlation of both IgG4 and IgE values with age.

In conclusion, this paper provides normal ranges for serum concentrations of IgG1, IgG2, and IgG3 which can be used by other laboratories, provided that standards are calibrated against the WHO serum and the assays are performed on frozen serum specimens. These normal ranges should help workers to assess the importance of low IgG subclass levels. 


\section{Appendix}

The following are the polynomial regressions fitted to the means and SDs of the IgG values, transformed where necessary. Age is in years.

(1) IgG1. Mean $=3.624+0.4428$ age; SD $=2 \cdot 166$ -0.3797 age +0.06274 age $^{2}$.

(2) Log (IgG2). Mean $=-0.7502+0.4377$ age +0.05667 age $^{2} ;$ SD $=0.541-0.09016$ age +0.01756 age $^{2}$.

(3) Log (IgG3). Mean $=-1 \cdot 16 ; \mathrm{SD}=0.4307$ -0.05073 age +0.009497 age $^{2}$.

\section{References}

' Morell A, Skvaril F, Steinberg AG, Loghem E-van, Terry WD. Correlations between the concentrations of the four sub-classes of $\mathrm{IgG}$ and $\mathrm{Gm}$ allotypes in normal human sera. J Immunol 1972;108:195-206.

${ }^{2}$ Schur PH, Borel M, Gelfand EW, Alper CA, Rosen FS. Selective gamma-G globulin deficiencies in patients with recurrent pyogenic infections. $N$ Engl $J$ Med 1970;283:631-4.

3 Oxelius V-A. Chronic infections in a family with hereditary deficiency of IgG2 and IgG4. Clin Exp Immunol 1974;17:19-27.

${ }^{4}$ Oxelius V-A, Laurell AB, Lindquist B, et al. IgG sub-classes in selective IgA deficiency: importance of IgG2-IGA deficiency. $N$ Engl J Med 1981;304:1476-7.

5 Oxelius V-A, Berkel AI, Hanson LA. IgG2 deficiency in ataxiatelangiectasia. $N$ Engl J Med 1982;306:515-7.

${ }^{6}$ Hammarström L, Smith CIE. IgG2 deficiency in a healthy blood donor. Concomitant lack of IgG2, IgA and IgE immunoglobulins and specific anti-carbohydrate antibodies. Clin Exp Immunol 1983;51:600-4.
${ }^{7}$ Isaacs D, Altman DG, Tidmarsh CE, Valman HB, Webster ADB. Serum immunoglobulin concentrations in preschool children measured by laser nephelometry: reference ranges for IgG, IgA and IgM. J Clin Pathol 1983;36:1193-6.

${ }^{8}$ Morell A, Skvaril F. A modified radioimmunoassay for quantitative determination of IgG sub-classes in man. In: Peeters H, ed. Protides of the biological fluids. Oxford: Pergamon Press, 1972.

${ }^{9}$ Gleich GJ, Averback AK, Svedlund HA. Measurement of IgE in normal and allergic serum by radioimmunoassay. $J$ Lab Clin Med 1971;77:690-8.

${ }^{10}$ Morell A, Skvaril F, Hitzig WH, Barandun S. IgG sub-classes: development of the serum concentrations in 'normal' infants and children. J Pediatr 1972;80:960-4

1 Schur PH, Rosen F, Norman ME. Immunoglobulin sub-classes in normal children. Pediatr Res 1979;13:181-3.

12 Oxelius V-A. IgG sub-class levels in infancy and childhood. Acta Paediatr Scand 1979;68:23-7.

13 Tidmarsh CE. Problems associated with the preparation of an immunoglobulin reference range by laser nephelometry. Thesis: Brunel University, 1980.

${ }^{14}$ French MAH, Harrison G. Serum IgG sub-class concentrations in healthy adults: a study using monoclonal antisera. Clin Exp Immunol 1984;56:473-5.

15 Hammarström L, Granström M, Oxelius V-A, Persson MAA. IgG sub-class distribution of antibodies against $S$. aureus teichoic acid and $\alpha$-toxin in normal and immunodeficient donors. Clin Exp Immunol 1984;55:593-601.

16 Riesen WF, Skvaril F, Braun DG. Natural infection of man with group A streptococci. Levels, restriction in class, sub-class, and type and clonal appearance of polysaccharide - group specific antibodies. Scand J Immunol 1976;5:383-90.

Correspondence to Dr A D B Webster, Division of Immunological Medicine, Clinical Research Centre, Harrow, Middlesex HA1 3UJ.

Received 19 December 1984 